\title{
High Resolution Prediction of Gas Injection Process Performance for Heterogeneous Reservoirs
}

\author{
Quarterly Report \\ July 1, 2003 to September 30, 2003 \\ Contract No. DE-FC26-00BC15319 \\ Principal Investigator: Franklin M. Orr, Jr. \\ Department of Petroleum Engineering \\ Stanford University
}

\section{Disclaimer}

This report was prepared as an account of work sponsored by an agency of the United States Government. Neither the United States Government nor any agency thereof, nor any of their employees, makes any warranty, express or implied, or assumes any legal liability or responsibility for the accuracy, completeness, or usefulness of any information, apparatus, product, or process disclosed, or represents that its use would not infringe privately owned rights. Reference herein to any specific commercial product, process, or service by trade name, trademark, manufacturer, or otherwise does not necessarily constitute or imply its endorsement, recommendation, or favoring by the United States Government or any agency thereof. The views and opinions of authors expressed herein do not necessarily state or reflect those of the United States Government or any agency thereof. 


\begin{abstract}
This report outlines progress in the first quarter of the extension of the DOE project "High Resolution Prediction of Gas Injection Process Performance for Heterogeneous Reservoirs".

This report presents experimental results that demonstrate combined scaling effects of viscous, capillary, and gravity crossflow mechanisms that apply to the situations in which streamline models are used.

We designed and ran a series of experiments to investigate combined effects of capillary, viscous, and gravity forces on displacement efficiency in layered systems. Analog liquids (isooctane, isopropanol, and water) were employed to control scaling parameters by changing interfacial tension (IFT), flow rate, and density difference. The porous medium was a twodimensional $(2-D)$ 2-layered glass bead model with a permeability ratio of about 1:4. In order to analyze the combined effect of only capillary and viscous forces, gravity effects were eliminated by changing the orientation of the glass bead model. We employed a commercial simulator, Eclipse100 to calculate displacement behavior for comparison with the experimental data.
\end{abstract}

Experimental results with minimized gravity effects show that the IFT and flow rate determine how capillary and viscous forces affect behavior of displacement. The limiting behavior for scaling groups for two-phase displacement was verified by experimental results. Analysis of the 2- $D$ images indicates that displacements having a capillary-viscous equilibrium give the best sweep efficiency. Experimental results with gravity effects, but with low IFT fluid systems show that slow displacements produce larger area affected by crossflow. This, in turn, enhances sweep efficiency. The simulation results represent the experimental data well, except for the situations where capillary forces dominate the displacement. 


\section{Contents}

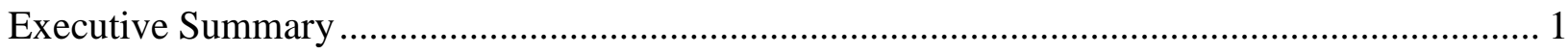

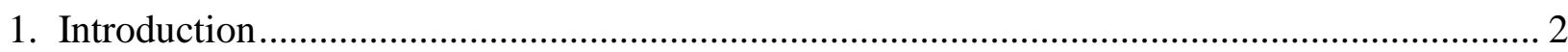

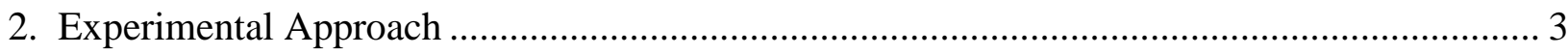

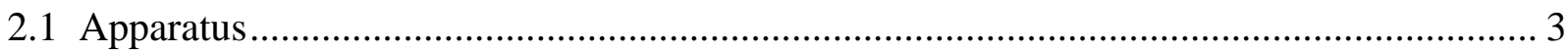

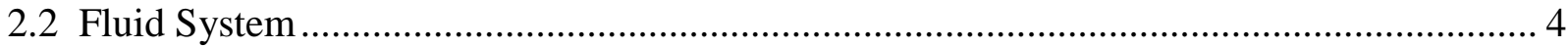

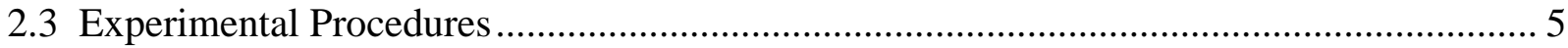

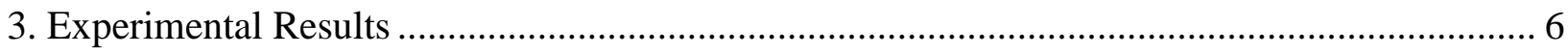

3.1 Homogeneity of the Flow Model............................................................................. 6

3.2 High-IFT Displacements with Reduced Effects of Gravity ....................................... 6

3.3 Low-IFT Displacements with Reduced Effects of Gravity ........................................... 7

3.4 Low-IFT Displacements with Gravity Effects ..................................................... 9

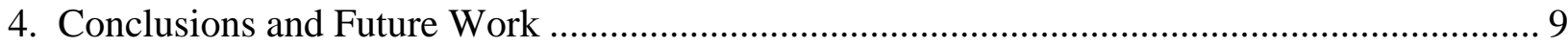

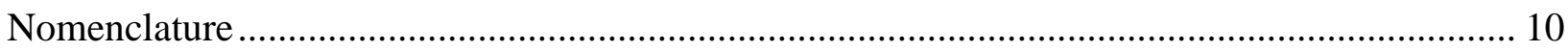

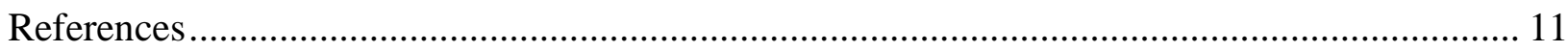

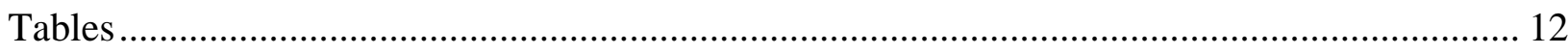

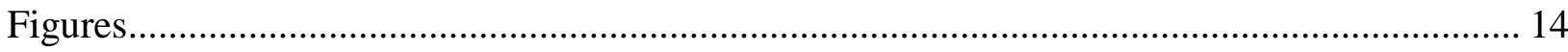




\section{Executive Summary}

While streamline methods offer many advantages for field-scale simulation, they are not without limitations. Because these methods treat flow along each streamline as independent of adjacent streamlines, effects of crossflow are not represented in the simulations. If users of streamline methods are to interpret simulation results reliably, they will need to assess whether any of the mechanisms not modeled in the simulations are important enough to limit appreciably the accuracy of the simulations.

We developed a strategy to investigate experimentally the combined effects of viscous, capillary, and gravity on displacement efficiency. Glass bead models were used to build a two-dimensional (2-D), two-layered flow model. The advantages of such models are that they allow the displacement to be directly visible, and that they enable us to have desired permeability heterogeneities. The high-permeability layer consisted of glass beads with a size of $0.05 \mathrm{~cm}$ yielded a permeability of $190 \mathrm{D}$ whereas the low-permeability layer packed with glass beads of $0.025 \mathrm{~cm}$ had $52 \mathrm{D}$. The porosity of both layers was measured to be $39.5 \%$. We oriented the models to allow gravity-stabilized displacements in the vertical direction. These displacements were used to evaluate combined effects of capillary and viscous crossflows. The homogeneity of the glass bead model was verified by miscible displacements. Two sets of overall phase compositions from the ternary isooctane $\left(I C_{8}\right)$ isopropanol-water system, in which interfacial tension (IFT) can be controlled systematically, were used. The first set of phases was composed of binary mixtures of $I C_{8}$ and water that represented High IFT in the experiments. The other one was composed of all ternary components, which represented Low IFT. The water-rich phase was injected to displace the $I C_{8}$-rich phase, a favorable displacement eliminating viscous instabilities. From the experiments, production and pressure data, 2- $D$ images of the displacement, and values of scaling factors were obtained. Eclipse100, a commercial black oil simulator, was used to simulate these experiments.

Experimental results of high-IFT displacements with reduced effects of gravity show that the IFT and flow rate determine how capillary and viscous forces affect behavior of displacement. The limiting behavior for scaling groups for two-phase displacement was verified by experimental results. It was determined from the 2- $D$ images that displacements having a capillary-viscous equilibrium should give the best sweep efficiency. Results of lowIFT experiments with gravity effects show that slow displacements have larger area affected by crossflow. It was observed experimentally that larger crossflow regions enhanced sweep efficiency. The simulation results represent the experimental data well, except for the situations where capillary forces dominate the displacement. 


\section{Introduction}

While streamline methods offer many advantages for field-scale simulation, they are not without limitations. Because these methods treat flow along each streamline as independent of adjacent streamlines, effects of crossflow are not represented in the simulations. If users of streamline methods are to interpret simulation results reliably, they will need to assess whether any of the mechanisms not modeled in the simulations are important enough to limit appreciably the accuracy of the simulations.

A key assumption in the streamline approach is that transfer of components across streamlines can be neglected. The scaling of diffusion and dispersion has been investigated in a variety of previous studies. If the injected gas is miscible or partially miscible with the oil, diffusion and dispersion mechanisms may play a significant role in the displacement [Jiang and Butler (1996), Fayers and Lee (1994), Tchelepi (1994), Mohanty and Johnson (1993)]. In particular, Burger and Mohanty (1997) showed that diffusion through the oil phase can limit mass transfer from oil residing in low permeability regions. Similar arguments can be applied to other mechanisms of crossflow: viscous crossflow, capillary crossflow, and gravity segregation [Burger and Mohanty (1997), Zhou et al. (1994), Fayers and Lee (1994)]. Experimental investigations at the short core scale have shown that crossflow effects can be significant in some settings, especially in heterogeneous media where high and low permeability zones exist [Schechter et al. (1994), Fayers and Lee (1994), Firoozabadi and Markeset (1994), Firoozabadi and Tan (1994), Burger and Mohanty (1997), Wylie and Mohanty (1997), Burger and Mohanty (1997), Ma et al. (997), Peters et al. (1998)]. What is needed now is a systematic analysis of the scaling of crossflow mechanisms applied to the situations in which streamline models are used.

Starting from material balance equations Zhou et al. (1994) presented scaling groups that determine the regime of flow during two-phase displacement. The relevant scaling groups are given as below:

$$
\begin{aligned}
& N_{g v}=\frac{\Delta \rho g L k_{a v}}{H v \mu_{o}} \\
& N_{c v}=\frac{L P_{c}^{*} k_{a v}}{H^{2} v \mu_{o}}
\end{aligned}
$$

Here, $N_{g v}$ and $N_{c v}$ are the characteristic time ratios for fluid to flow in the transverse direction due to gravity or capillary forces to that in horizontal direction due to viscous forces ${ }^{*}$. The $P_{c}^{*}$ is a characteristic transverse capillary pressure of the medium, which is defined by,

$$
P_{c}^{*}=\int_{S_{w c}}^{1-S_{o r}} \frac{p_{c}\left(S_{w}\right)}{\left(1-S_{o r}-S_{w c}\right)} d S
$$

Zhou et al. reported criteria that determine when capillary forces become dominant in the displacement as well as when gravity forces or viscous forces do. They defined the transients

\footnotetext{
*All other symbols are defined in the nomenclature section.
} 
between them as equilibrium conditions such as gravity-capillary, viscous-capillary, and viscous-gravity equilibrium. Table 1 summarizes related scaling factors and dominated regions. The $M$ in the table is the mobility ratio:

$$
M=\frac{\lambda_{w}}{\lambda_{o}} \approx \frac{\mu_{o}}{\mu_{w}}
$$

While several components of the crossflow process have been investigated, a full set of experiments designed to examine individually and simultaneously the effects of capillary, diffusion, dispersion, gravity, and viscous forces in two- and three-phase miscible (or partially miscible) gas injection has not been carried out. Experimental work carried out so far basically includes miscible displacements in 2- $D$ glass bead models. Such models allow to visualize displacement fronts as well as the area affected by crossflow.

Brock and Orr (1991) investigated the combined effect of viscous fingering and permeability heterogeneity. They employed four different glass bead models with different permeability heterogeneities, which were made by packing unconsolidated glass beads in a quasi-2- $D$ linear geometry. They used first-contact miscible fluids in the experiments to eliminate capillary effects. They controlled the effect of viscous forces with different flow rates. They employed particle tracking simulations of unstable flows to simulate experiments. In homogeneous media, viscous fingers grow by spreading and splitting at their tips. In heterogeneous media fingering patterns develop along the same streamlines followed during flow at unit mobility in the same porous medium. In general, simulations reproduced the fingering patterns of the experiments well. However, the experimental fingers were more diffuse at their tips than were fingers in the simulations, probably due to some edge flow of fluids in the experimental models.

Peters et al. (1998) worked out the effect of gravity on miscible displacements. They performed a series of experiments in two layered glass bead systems where gravity forces favored flow in the low-permeability layer. They observed that increasing the gravity number forced fluid from the high-permeability layer into the low-permeability layer, increasing crossflow and improving recovery. They also stated that, for the favorable mobility (mobility ratio, $M<1$ ), viscous and gravity crossflow forces opposed each other whereas they acted in the same direction for the unfavorable mobility $(M>1)$.

In this report we present experimental results that demonstrate effects of capillary, viscous, and gravity crossflow on two-phase displacement.

\section{Experimental Approach}

\subsection{Apparatus}

Glass bead models were used to build a two-dimensional, two-layered flow model. Such models allow the displacement to be directly visible, and they enable control permeability heterogeneities. A schematic of the apparatus used in this study is shown in Fig. 1. 
The glass bead model was the key part of the apparatus. The model was built in the following way (see Fig. 2): First, glass blocks and fluid distribution parts were glued together with epoxy to form a box with one side open. This box was then attached to two pneumatic vibrators. As a first, small-sized glass beads (Mesh size: 60 , bead size $\approx 0.025 \mathrm{~cm}$ ), and then large glass beads (Mesh size: 35 , bead size $\approx 0.05 \mathrm{~cm}$ ) were dropped into the model under vibration. After that some water was let into the model to make sure have a better compaction of glass beads and then the packing was pressed firmly by means of a plastic plate. The final piece of the box was then glued in place by applying an additional forced on that piece. The water was drained and the model was flushed by dry air for 24 hours.

The porosity of each layer was measured separately, and found to be approximately $39.5 \%$. The permeability of each layer was also measured separately using homogeneous packs in a cylindrical tube: $190 \mathrm{D}$ for high-permeability layer and $52 \mathrm{D}$ for low-permeability layer. Using the individual permeabilities, the average permeabilities of the model in the horizontal and vertical directions can be calculated by the following formulas:

$$
\begin{aligned}
& k_{a h}=\frac{\sum_{i} k_{i} H_{i}}{\sum_{i} H_{i}} \\
& k_{a v}=\frac{\sum_{i} H}{\sum H_{i} / k_{i}}
\end{aligned}
$$

where the $H$ represents the height of the layer. The calculated average permeabilities are 83 $D$ and $121 D$ for vertical and horizontal directions, respectively. The horizontal permeability of the model was verified experimentally. We prepared short and long models to investigate effects of gravity.

Pressure drop across the model and production of the phases at the end of the model were measured during the experiment. The pressure was measured by means of an analog gauge with an accuracy of $0.2 \mathrm{kPa}$. The effluent fluids were collected in plastic graduated cylinders. After suitable delay for phase separation, the production data were then determined by reading the liquid levels in the cylinders with an accuracy of $0.1 \mathrm{~cm}^{3}$. All displacements were recorded using a video camera to obtain areas invaded across the model.

We oriented the models to allow gravity-stabilized displacements in the vertical direction. These displacements were used to evaluate combined effects of capillary and viscous crossflows.

\subsection{Fluid System}

We employed two different sets of overall phase compositions from the ternary isooctane $\left(I C_{8}\right)$-isopropanol (IPA)-water system, in which IFT can be controlled systematically (Fig. 3). The first set of phases was composed of binary mixture of $I C_{8}$ and water that represented 
High IFT in the experiments. The other one was composed of all ternary components, which represented Low IFT in this study. The fluid properties of the phases are given in Table 2.

\subsection{Experimental Procedures}

Table 3 gives a preliminary strategy for all experiments. We used the following steps to investigate the combined effects of crossflow forces:

- We employed two different glass bead models (one short, one long) to investigate $L / H$ ratios on crossflow formed by gravity forces.

- We employed gravity-stabilized experiments to investigate the combined effects of only viscous and capillary forces.

- We injected fluids with three different flow rates to control viscous forces.

- We used one low and one high IFT to study the effect of capillary crossflow with/out gravity forces.

- In future experiments, we will repeat some of the experiments with an unfavorable mobility ratio.

For the experiments with gravity, the model was located such that the low-permeability layer was at the bottom. Later we inverted the glass bead model such that the high-permeability layer was at the bottom and repeated some of the experiments.

In this report we only present the experimental results with favorable mobility ratio. In the second phase of the experiments, we will perform displacements with unfavorable mobility.

We basically employed the following experimental procedure:

i. Displace air in the pores with $\mathrm{CO}_{2}$

ii. Displace the $\mathrm{CO}_{2}$ with two $P V \mathrm{~s}$ of the dyed $I C_{8}$-rich phase to make sure that the porous system is saturated completely.

iii. Then start injecting the dyed $\mathrm{H}_{2} \mathrm{O}$-rich phase to view the saturation profile using Camcorder. Record the pressure drop across the model and the production of phases.

iv. Compare the experimental results with simulation results.

At the beginning of experiments, we tried to create a residual saturation of the $\mathrm{H}_{2} \mathrm{O}$-rich phase in the porous system to represent displacements in real reservoirs. However, after preliminary test runs, we determined that we could not obtain a reasonable residual saturation of the $\mathrm{H}_{2} \mathrm{O}$-rich phase for the porous system. This was because the injected $\mathrm{IC}_{8}$-rich phase did not sweep the water-rich phase in the low-permeability layer after breakthrough in the high-permeability layer. We separately measured a residual water saturation of about $10 \%$ for glass bead packs. In the end, we skipped the steps for the residual saturation and performed experiments of the $\mathrm{H}_{2} \mathrm{O}$-rich phase displacing the $\mathrm{IC}_{8}$-rich phase fully saturated in pores. 


\section{Experimental Results}

\subsection{Homogeneity of the Flow Model}

The homogeneity of the flow model was checked by the following experiment: The model was first saturated completely by water. Then first-contact miscible dyed water was injected to the porous medium. Because the fluids were miscible and there were no capillarity and density difference, the effects of capillary and gravity forces on the displacement were eliminated. The displacement fronts in each layer should then be determined by the individual permeabilities. The experimental results are shown in Fig. 4. The model was held vertically and all fluids were injected from bottom. The first image shows the situation at the start of the experiment, whereas the second shows the saturation profile at the time the pore volume injected $(P V I)$ was 0.35 . The displacement front in the high-permeability layer (at $x \sim 29 \mathrm{~cm}$ of the total length of $54 \mathrm{~cm})$ is faster than that in the low-permeability layer $(x \sim 9$ $\mathrm{cm}$ ). The ratio of the front locations (about 3.2) is comparable to the permeability ratio (3.7). The third image shows the saturation distribution in the layers at the breakthrough. The images prove an acceptable homogeneity of the individual layers.

\subsection{High-IFT Displacements with Reduced Effects of Gravity}

We employed vertical displacements with the flow rates of $0.6,2.3$, and $8.5 \mathrm{~cm}^{3} / \mathrm{min}$. The related crossflow scaling groups for these experiments were calculated using Eqs. 1 and 2. A summary of data and calculations is given in Table 4. The so-called characteristic capillary pressure, $P_{c}^{*}$, is evaluated from the capillary pressure experimental data using the correlation developed by Brooks and Corey (1966). Using the Brooks-Corey correlation in Eq. 3 we have,

$$
P_{c}^{*}=\int_{o}^{1} p_{d}\left(S_{w d}\right)^{-1 / \lambda} d S_{w d}
$$

Where,

$$
S_{w d}=\frac{S_{w}-S_{w c}}{1-S_{w c}-S_{o r}}
$$

The $p_{d}$ and $\lambda$ can be determined using experimental capillary pressure data. We used the data of Leverett $J$-function, presented by Collins (1961) for unconsolidated sand packs for waterkerosene fluid system. Taking $S_{w c}=0.1$ and $S_{o r}=0$ we obtained $\lambda=5$ and $p_{d}=0.5$ and $800 P a$ for low and high IFT, respectively (see the correlations in Fig. 5).

We also used Eclipse100, a commercial black oil simulator, to simulate experiments. The same $J$-function and the relative permeability shown in Fig. 6 were used as input data for the simulator. The measured data in Fig. 6 were obtained using bead packs and Hexadecanewater fluid system. The correlated data were obtained using the correlation developed by Corey (1954). 
All three displacements had capillary numbers that are greater than unity, accordingly the flow mechanism controlling the sweep efficiency was clearly capillary-dominated. However, the displacement profiles shown in Fig. 7 prove that the effects of capillary forces on the flow decrease dramatically as the flow rate is increased. The images show the $\mathrm{H}_{2} \mathrm{O}$-rich phase (red) displacing the $I C_{8}$-rich phase (light green) with no irreducible water in the porous system. In the first set of images at $P V I=0.19$, there is a clear capillary interaction to be seen at the interface between the layers. At the slow flow rate, the displacement front in the lowpermeability layer is faster than that in the high-permeability layer because of longitudinal capillary imbibition. The front looks like a tongue along the interface. Because of capillary end effects, the injected phase cannot crossflow into the high-permeability layer. The sweep efficiency, therefore, is lower than at the faster velocities, where longitudinal capillary imbibition is too slow to create a leading tongue in the low permeability layer. If the displacement had been miscible, we would have observed faster flow in the highpermeability layer and viscous crossflow at the interface. As the flow rate is increased by four, the front in the low-permeability layer slows down whereas the front in the highpermeability layer moves faster, which indicates that the effects of viscous forces increase in magnitude compared to the effects of the capillary forces. The leading edge of the displacement front is still tongue-like but smaller. As the flow rate is increased one more time by a factor of four, the displacement fronts in both layers stabilize as can be seen from Fig. 7. The capillary number still shows that capillary forces can dominate the flow, however, a capillary-viscous equilibrium can also be interpreted from that image. Compared to previous two images, a better sweep efficiency can be seen in this case. At later stages of the displacement, i.e. at $P V I=0.56$, similar displacement profiles were obtained for the relevant flow rates. After one $\mathrm{PV}$ of the $\mathrm{H}_{2} \mathrm{O}$-rich phase was injected, it was observed that the sweep efficiency at the situation that capillary forces dominate due to the effects of longitudinal capillary imbibition was worse than the others did. However, almost the same amount of the $I C_{8}$-rich phase was recovered for each case after $P V I=1$.

Fig. 8 compares the production and pressure profiles for the experiments and corresponding simulation. In general the simulation results agreed very well with the production profiles except for the pressure data. The simulated pressure is the difference between the block pressures at the first and last blocks in the vertical direction. The experimental pressure was it measured at the entrance outside the porous model. This could result in some differences in the simulated and measured pressures. Especially at the situations where capillary forces were strongly dominant, the simulation predictions for the pressure drop are far from the error limits of experimental data (Some convergence problems were observed in the simulations at low rate, as the oscillations in the calculated pressures show). As the effect of capillary forces decreased (see Fig. 8c), the simulator gave better predictions for the pressure drop. The simulated recovery values at $P V I=1$ are approximately comparable with experimental data.

\subsection{Low-IFT Displacements with Reduced Effects of Gravity}

The calculated scaling groups and relevant data are given in Table 5. A relatively low characteristic capillary pressure (because of the low IFT) results in low capillary numbers. It is, therefore, expected that viscous forces should be dominant in the displacement. 
Experiments were performed in the same way as explained above. The images taken at different $P V I$ values during the experiment are shown in Fig. 9. In this experiment, the injected $\mathrm{IC}_{8}$-rich fluid was dyed green, and the $\mathrm{H}_{2} \mathrm{O}$-rich fluid was red. The first impression from the images is that the displacement fronts are totally different from those obtained with high-IFT fluid system. This can be explained by the fact that the three-order of magnitude reduction in IFT (from 38.1 to $0.024 \mathrm{mN} / \mathrm{m}$ ) reduces the capillary pressure by the same magnitude (see Fig. 6).

Although we also reduced the effects of gravity by performing gravity-stabilized vertical displacements, the difference between the fronts of the fast and slow layers leads to some effect of gravity on displacement performance in such systems. To show the magnitude of this effect, we ran the simulator with different values for density differences (see Fig. 10). The figure shows that an increase in density difference of the phases causes the front in the fast layer to slow down, whereas it advances the slow layer. This effect results in an improved sweep of the porous medium. However, in the related experiment where the density difference was about $0.07 \mathrm{~g} / \mathrm{cm}^{3}$, gravity affects on the displacement performance were small.

Since gravity and capillary effects were minimized by lowering IFT and employing gravitystabilized vertical displacements, the front position in each layer is determined by viscous forces as well as the level of communication between the layers. If there is no communication between the layers, the front position in each layer may be determined from Darcy's law [Lake (1989)]. However, communication between layers leads to crossflow due to the different pressure gradients in the layers driven by viscous and gravity forces. Zapata and Lake (1981) presented a theoretical analysis of viscous crossflow in layered reservoirs with absence of capillary and gravity effects. They explained viscous crossflow in both favorable and unfavorable displacements starting from pressure profiles for two noncommunicating layers. They concluded that for favorable displacements the direction of crossflow is from the low to high permeability layer at the leading water front and in the reverse direction at the trailing water front. Thus crossflow causes the leading and trailing fronts to recede and advance, respectively, over their no-crossflow positions. This, in turn, improves the vertical sweep efficiency over that of the situation without crossflow.

Fig. 11 shows an explanation schematic for one of the reported favorable displacements in terms of the pressure profiles. Neglecting the effects of capillary pressure (a reasonable assumption for the low IFT of $0.024 \mathrm{mN} / \mathrm{m}$ ) and assuming no communication between the layers, we can consider viscous and gravity forces to determine the pressure profile along the porous medium. The gravity forces slightly increase the rate of crossflow depending on the density difference as shown in Fig.10. Applying the pressure profiles in a communicating layer system, we have two different crossflows in the system. The first one takes place at the leading front and its direction is from the slow layer into the fast layer. And the second one is the crossflow from fast layer into the slow layer at the trailing front. The displacement profile obtained experimentally is clearly consistent with the explanation given. 
It is also interesting to note that when we compare the images at $P V I=0.31$ with those from Fig. $7(P V I=0.19)$ in Fig. 12. We clearly see the effects of the transitions from capillary to viscous dominated flow on the displacement profile. Providing that gravity forces are small, an equilibrium between capillary and viscous forces gives an optimum sweep efficiency for the displacement defined in this section.

Experimental and simulation results for the production and pressure drop are given in Fig. 13. Because the displacements were run under the stability of viscous and gravity forces, there were no big differences obtained in the recoveries. The simulation results represent the experimental production and pressure data very well. This is mainly because IFT is the lower, which reduces the effects of capillary pressure.

\subsection{Low IFT Displacements with Gravity Effects}

The effects of gravity were included in these experiments by the way that the glass bead model was oriented horizontally. The high-permeability layer was at the top of the model. The scaling parameters for these experiments were calculated and are given in Table 6 . We used favorable displacements to avoid viscous instabilities in the experiments. The low-IFT liquid system was employed, so capillary effects were small (See the low modified capillary numbers in Table 6).

Based on the criteria given in Table 1, we argue that the low-rate displacement should show the largest gravity effects. But, this effect becomes weaker as the rate is increased. For the intermediate rate experiment we can expect that an equilibrium between gravity and viscous forces might establish. The last experiment with the high flow rate will certainly be controlled by viscous forces.

Fig. 14 shows the images that illustrate the crossflow regions in the low-permeability layer. Two gravity tongues can be distinguished clearly on the images of the low rate experiment. It is expected that the gravity tongue in the top high-permeability layer would be longer than the bottom one. However, the crossflow that occurs from the upper layer into the lower layer makes the upper tongue smaller. With increasing the flow rate the gravity tongue tongues in both layers disappear. The fronts in both layers look more stabilized with a small crossflow region. As it can be seen from the images, the highest sweep efficiency is found for the lowrate displacement, which is affected by gravity. Thus, for this combination of displacement and flow model, the crossflow increases the ultimate sweep efficiency.

Fig. 15 shows production and pressure profiles for the experiments. Simulation results agree with the experimental data.

\section{Conclusions and Future Work}

A series of experiments was designed and run to investigate combined effects of capillary, viscous, and gravity forces on displacement efficiency in layered systems. Two-phase displacements with favorable mobility ratio were performed. 
The high-IFT experimental results with small gravity effects show that the IFT and flow rate determine how capillary and viscous forces affect behavior of displacement. The limiting behavior for scaling groups presented by Zhou et al. (1994) was verified by the experimental results. High values of $N_{c v}$, capillary forces caused the displacement front to move more rapidly in the low-permeability layer. In contrast at low values of $N_{c v}$, the front moved more rapidly in the high-permeability layer. The 2-D images show that displacements in which capillary and viscous forces are roughly balanced give displacement fronts that move at comparable velocities in both low- and high-permeability layers, a situation that results in the optimum sweep efficiency.

The results of the low-IFT experiments with gravity effects show that slow displacements produce larger area affected by crossflow. Consequently, it contributes positively to the total sweep efficiency of the porous system.

Numerical simulation of the displacements matches the experimental production history and sweep efficiencies very well, except for the observed and calculated pressure drops in the situations where capillary forces dominate the displacement.

The future work includes the rest of the work defined in Table 3, namely, experiments with (1) gravity, viscous and capillary effects, (2) unfavorable viscosity ratio, (3) the glass model having the low-permeability layer at the top, and (4) a long glass bead model.

\section{Nomenclature}

$\begin{array}{ll}g & \text { gravitational force } \\ H & \text { height of the layers } \\ k_{a h} & \text { horizontal permeability of the model } \\ k_{a v} & \text { vertical permeability of the model } \\ k_{i} & \text { permeability of layer i } \\ L & \text { length of the model } \\ M & \text { viscosity ratio } \\ N_{c v} & \text { modified capillary number } \\ N_{g v} & \text { modified gravity number } \\ P_{c} * & \text { characteristic capillary pressure } \\ p_{c} & \text { capillary pressure } \\ p_{d} & \text { displacement pressure } \\ S_{o r} & \text { residual oil saturation } \\ S_{w c} & \text { residual water saturation } \\ q & \text { flow rate } \\ v & \text { Darcy velocity } \\ W & \text { width of the model } \\ \mu_{o} & \text { oil viscosity } \\ \phi & \text { porosity } \\ \Delta \rho & \text { density difference } \\ I F T & \text { interfacial tension } \\ I P A & \text { isopropanol } \\ P V I & \text { pore volume injected }\end{array}$




\section{References}

1. Burger, J.E. and Mohanty, K.K.: Mass transfer from bypassed zones during gas injection. Soc. Pet. Eng. Reservoir Engineering, 12 (2), 124-130, 1997.

2. Fayers, J.J. and Lee, S.T.: Crossflow mechanisms in oil displacement by gas drive in heterogeneous reservoirs. In Situ, 18 (4), 369-398, 1994.

3. Firoozabadi, A. and Markeset, T.: Miscible displacement in fractured porous media: Part I Experiments. SPE/DOE 27743, in Proc. of the SPE/DOE Improved Oil Recovery Symposium, Tulsa, OK, April, 1994.

4. Firoozabadi, A. and Tan, J.C.T.: Miscible displacement in fractured porous media: Part II Analysis. SPE/DOE 27743, in Proc. of the SPE/DOE Improved Oil Recovery Symposium, Tulsa, OK, April, 1994.

5. Jiang, Q. and Butler, R.M.: Experimental studies on effects of reservoir heterogeneity on the Vapex process. J. Canadian Pet. Tech., 35 (10), 46-54, 1994.

6. Ma, S.X., Morrow, N.R., and Zhang, X.Y.: Generalized scaling of spontaneous imbibition data for strongly water-wet systems. J. Pet. Sci. Eng., 18 (3-4), 165-178, 1997.

7. Mohanty, K.K. and Johnson, S.W.: Interpretation of laboratory gasfloods with multidimensional compositional modeling. Soc. Pet. Eng. Reservoir Engineering, 8 (1), 59-66, 1993.

8. Peters, B.M., Zhou, D. and Blunt, M.J.: Experimental investigation of scaling factors that describe miscible floods in layered systems. SPE/DOE 39624, in Proc. of the SPE/DOE Improved Oil Recovery Symposium, Tulsa, OK, April, 1998.

9. Schechter, D.S., Zhou, D., and Orr, F.M., Jr.: Low IFT drainage and imbibition. J. Pet. Sci. Eng., 11, 283-300, 1994.

10. Tchelepi, H.A.: Viscous fingering, gravity segregation and permeability heterogeneity in twodimensional and three-dimensional flows. Ph.D. thesis, Stanford University, March, 1994.

11. Zhou, D., Fayers, F.J. and Orr, F.M., Jr.: Scaling of multiphase flow in simple heterogeneous porous media. SPE/DOE 27833, in Proc. of the SPE/DOE Improved Oil Recovery Symposium, Tulsa, OK, April, 1994.

12. Wylie, P. and Mohanty, K.K.: Effect of water saturation on oil recovery by near-miscible gas injection. SPE Reservoir Engineering, 21 (4), 264-268, 1997.

13. Brock, D.C. and Orr, F.M., Jr.: Flow visualization of viscous fingering in heterogeneous porous media. SPE 22614, in Proc. of the $66^{\text {th }}$ ATCE of the Soc. Pet. Tech., Dallas, TX, 1991.

14. Brooks, R.H. and Corey, A.T.: Properties of porous media affecting fluid flow. J. Irrig. Drain. Div., 6, 61, 1966.

15. Collins, R.E.: Flow of fluids through porous materials. Reinhold Publishing Corp., N.Y., 1961.

16. Corey, A.T.: The interrelation between gas and oil relative permeabilities. Prod. Montly, 19, 38, 1954.

17. Lake, L.: Enhanced Oil Recovery. Prentice Hall, Englewood Cliffs, New Jersey, 1989.

18. Zapata, V.J. and Lake, L.: A theoretical analysis of viscous crossflow. SPE 10111, presented at the $56^{\text {th }}$ Annual Tech. Conf. and Exhib., San Antonio, TX, Oct. 3-7, 1981. 
Table 1 - Conditions for flow regions [Zhou et al. (1994)].

\begin{tabular}{|l|l|}
\hline Flow Region & Condition \\
\hline Capillary-dominated & $N_{c v} \gg N_{g v}$ and $\frac{M N_{c v}}{1+M} \gg>1.0$ \\
\hline Gravity-dominated & $N_{g v} \gg N_{c v}$ and $\frac{M N_{g v}}{1+M} \gg>1.0$ \\
\hline Capillary-gravity equilibrium & $N_{c v} \approx N_{g v}$ and $\frac{M N_{g v}}{1+M}>>1.0$ \\
\hline Viscous-dominated & $\frac{M\left(N_{c v}+N_{g v}\right)}{1+M}<<1.0$ \\
\hline
\end{tabular}

Table 2 - Physical properties of the phases.

\begin{tabular}{|l|c|c|c|c|}
\hline & \multicolumn{2}{|c|}{ Tie-line 1 (Fig. 3) } & \multicolumn{2}{c|}{ Tie-line 2 (Fig. 3) } \\
\hline & $I C_{8}$-rich & $\mathrm{H}_{2} \mathrm{O}$-rich & $I C_{8}$-rich & $\mathrm{H}_{2} \mathrm{O}$-rich \\
\hline$\rho, g / \mathrm{cm}^{3}$ & 0.692 & 0.998 & 0.723 & 0.795 \\
\hline$\mu, m P a . s$ & 0.48 & 1 & 0.836 & 2.027 \\
\hline$I F T, m N / m$ & \multicolumn{2}{|c|}{38.1} & \multicolumn{2}{c|}{0.024} \\
\hline$\Delta \rho, g / \mathrm{cm}^{3}$ & \multicolumn{2}{|c|}{0.306} & \multicolumn{2}{c|}{0.072} \\
\hline$M\left(\mathrm{H}_{2} \mathrm{O}\right.$-rich disp. $I C_{8}$-rich $)$ & \multicolumn{2}{|c|}{0.48} & \multicolumn{2}{c|}{0.41} \\
\hline$M\left(I C_{8}\right.$-rich disp. $\mathrm{H}_{2} \mathrm{O}$-rich $)$ & \multicolumn{2}{|c|}{2.48} & \multicolumn{2}{|c|}{2.44} \\
\hline
\end{tabular}

Table 3 - Preliminary strategy for the displacement experiments.

\begin{tabular}{|c|c|c|c|c|c|c|}
\hline \multirow{2}{*}{ Glass Bead Model } & \multirow{2}{*}{$\begin{array}{l}\text { Gravity } \\
\text { Effects }\end{array}$} & \multirow{2}{*}{$\begin{array}{c}q \\
\mathrm{~cm}^{3} / \min \end{array}$} & \multicolumn{2}{|c|}{$\begin{array}{c}\text { Favorable displacement } \\
\qquad M<1\end{array}$} & \multicolumn{2}{|c|}{$\begin{array}{l}\text { Unfavorable displacemet } \\
\qquad M>1\end{array}$} \\
\hline & & & $\begin{array}{c}38.1 \mathrm{mN} / \mathrm{m} \\
M=0.48\end{array}$ & $\begin{array}{c}0.02 \mathrm{mN} / \mathrm{m} \\
M=0.41\end{array}$ & $\begin{array}{c}38.1 \mathrm{mN} / \mathrm{m} \\
M=2.08\end{array}$ & $\begin{array}{c}0.02 \mathrm{mN} / \mathrm{m} \\
M=2.44\end{array}$ \\
\hline \multirow{6}{*}{$\begin{array}{l}\text { Short }(34 \mathrm{~cm}) \text {, High- } \\
\text { perm layer at the top }\end{array}$} & \multirow{3}{*}{ Reduced } & 0.6 & • & • & & \\
\hline & & 2.2 & • & • & - & • \\
\hline & & 8.5 & $\bullet$ & - & & \\
\hline & \multirow{3}{*}{ large } & 0.6 & $\bullet$ & $\bullet$ & & \\
\hline & & 2.2 & $\bullet$ & $\bullet$ & $\bullet$ & $\bullet$ \\
\hline & & 8.5 & - & • & & \\
\hline $\begin{array}{l}\text { Long }(54 \mathrm{~cm}), \text { High- } \\
\text { perm layer at the top }\end{array}$ & large & 2.2 & $\bullet$ & $\bullet$ & $\bullet$ & $\bullet$ \\
\hline $\begin{array}{l}\text { Short, High-perm } \\
\text { layer at the bottom }\end{array}$ & large & 2.2 & $\bullet$ & $\bullet$ & $\bullet$ & • \\
\hline
\end{tabular}


Table 4 - Summary of experiments with HIGH IFT and REDUCED GRAVITY crossflow.

\begin{tabular}{|l|l|l|l|}
\hline$I F T, m N / m$ & \multicolumn{3}{|l|}{38.1} \\
\hline$M$ & \multicolumn{3}{|l|}{0.48 (Favorable displacement) } \\
\hline$q, \mathrm{~cm}^{3} / \mathrm{min}$ & 0.6 & 2.3 & 8.5 \\
\hline$N_{c v}$ & 480 & 118 & 32 \\
\hline$M N_{c v} /(1+M)$ & 156 & 38 & 11 \\
\hline $\begin{array}{l}\text { Recovery at } P V I=1- \\
\text { Experimental }\end{array}$ & 0.897 & 0.902 & 0.907 \\
\hline $\begin{array}{l}\text { Recovery at } P V I=1- \\
\text { Eclipse100 }\end{array}$ & 0.871 & 0.845 & 0.915 \\
\hline
\end{tabular}

\begin{tabular}{|l|l|}
\hline$L, c m$ & 33 \\
\hline$H, c m$ & 8 \\
\hline$W, c m$ & 0.65 \\
\hline$\mu_{o}, m P a . s$ & 0.48 \\
\hline$k_{a v}, D$ & 82 \\
\hline$P_{c}^{*}, P a$ & 1000 \\
\hline
\end{tabular}

Table 5 - Summary of experiments with LOW IFT and REDUCED GRAVITY crossflow.

\begin{tabular}{|l|l|l|l|}
\hline$I F T, m N / m$ & \multicolumn{3}{|l|}{0.024} \\
\hline$M$ & \multicolumn{3}{|l|}{0.41 (Favorable displacement) } \\
\hline$q, \mathrm{~cm}^{3} / \mathrm{min}$ & 0.6 & 2.1 & 8.5 \\
\hline$N_{c v}$ & 0.16 & 0.047 & 0.012 \\
\hline$M N_{c v} /(1+M)$ & 0.05 & 0.014 & 0.003 \\
\hline $\begin{array}{l}\text { Recovery at } P V I=1- \\
\text { Experimental }\end{array}$ & 0.922 & 0.884 & 0.901 \\
\hline $\begin{array}{l}\text { Recovery at } P V I=1- \\
\text { Eclipse100 }\end{array}$ & 0.955 & 0.914 & 0.862 \\
\hline
\end{tabular}

\begin{tabular}{|l|l|}
\hline$P_{c}^{*}, P a$ & 0.625 \\
\hline
\end{tabular}

Table 6 - Summary of experiments with LOW IFT and GRAVITY crosslow.

\begin{tabular}{|l|l|l|l|}
\hline$I F T, m N / m$ & \multicolumn{3}{|l|}{0.024} \\
\hline$\Delta \rho, g / \mathrm{cm}^{3}$ & \multicolumn{3}{|l|}{0.072} \\
\hline$M$ & \multicolumn{4}{|l|}{0.41 (Favorable displacement) } \\
\hline$q, \mathrm{~cm}^{3} / \mathrm{min}$ & 0.6 & 2.2 & 8.9 \\
\hline$N_{c v}$ & 0.17 & 0.045 & 0.011 \\
\hline$M N_{c v} /(1+M)$ & 0.05 & 0.018 & 0.003 \\
\hline$N_{g v}$ & 15.6 & 4.02 & 0.99 \\
\hline$M N_{g v} /(1+M)$ & 4.57 & 1.17 & 0.288 \\
\hline$M\left(N_{c v}+N_{g v}\right) /(1+M)$ & 4.61 & 1.19 & 0.294 \\
\hline $\begin{array}{l}\text { Recovery at } P V I=1- \\
\text { Experimental }\end{array}$ & 0.922 & 0.906 & 0.919 \\
\hline $\begin{array}{l}\text { Recovery at } P V I=1- \\
\text { Eclipse } 100\end{array}$ & 0.929 & 0.914 & 0.869 \\
\hline
\end{tabular}

\begin{tabular}{|l|l|}
\hline$P_{c}^{*}, P a$ & 0.625 \\
\hline
\end{tabular}




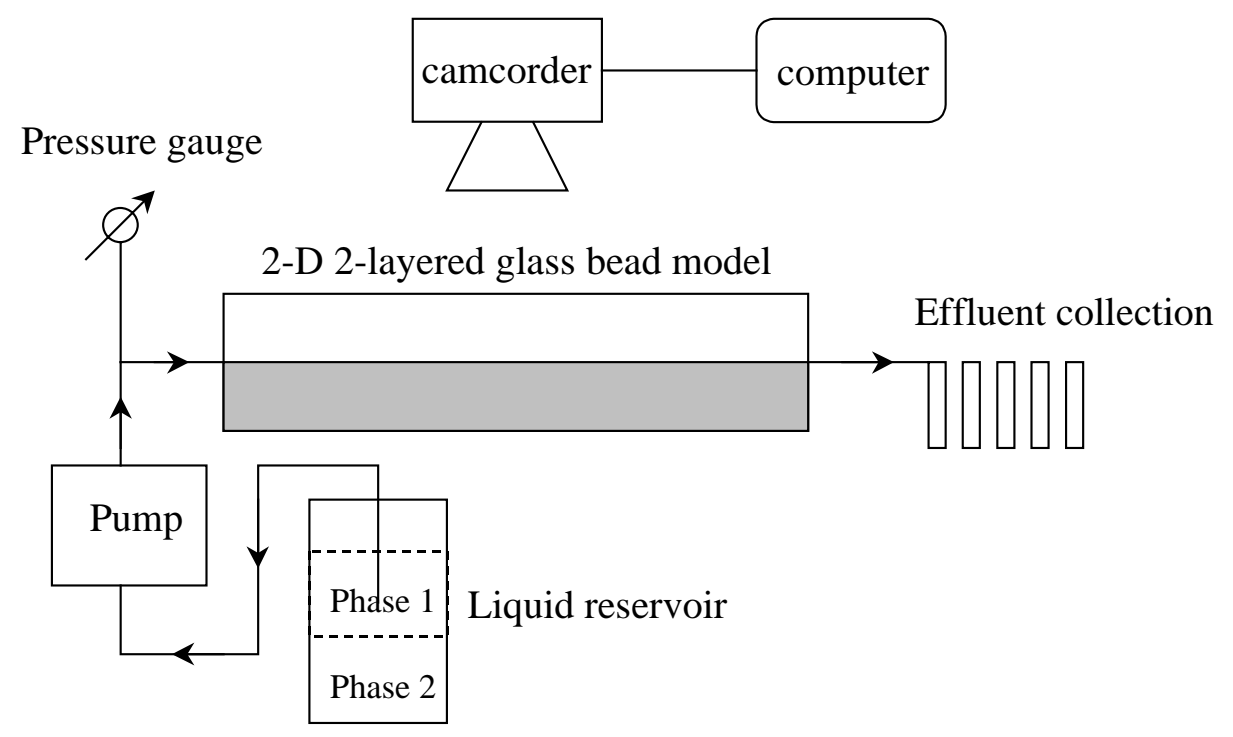

Figure 1 - A schematic of the experimental setup.
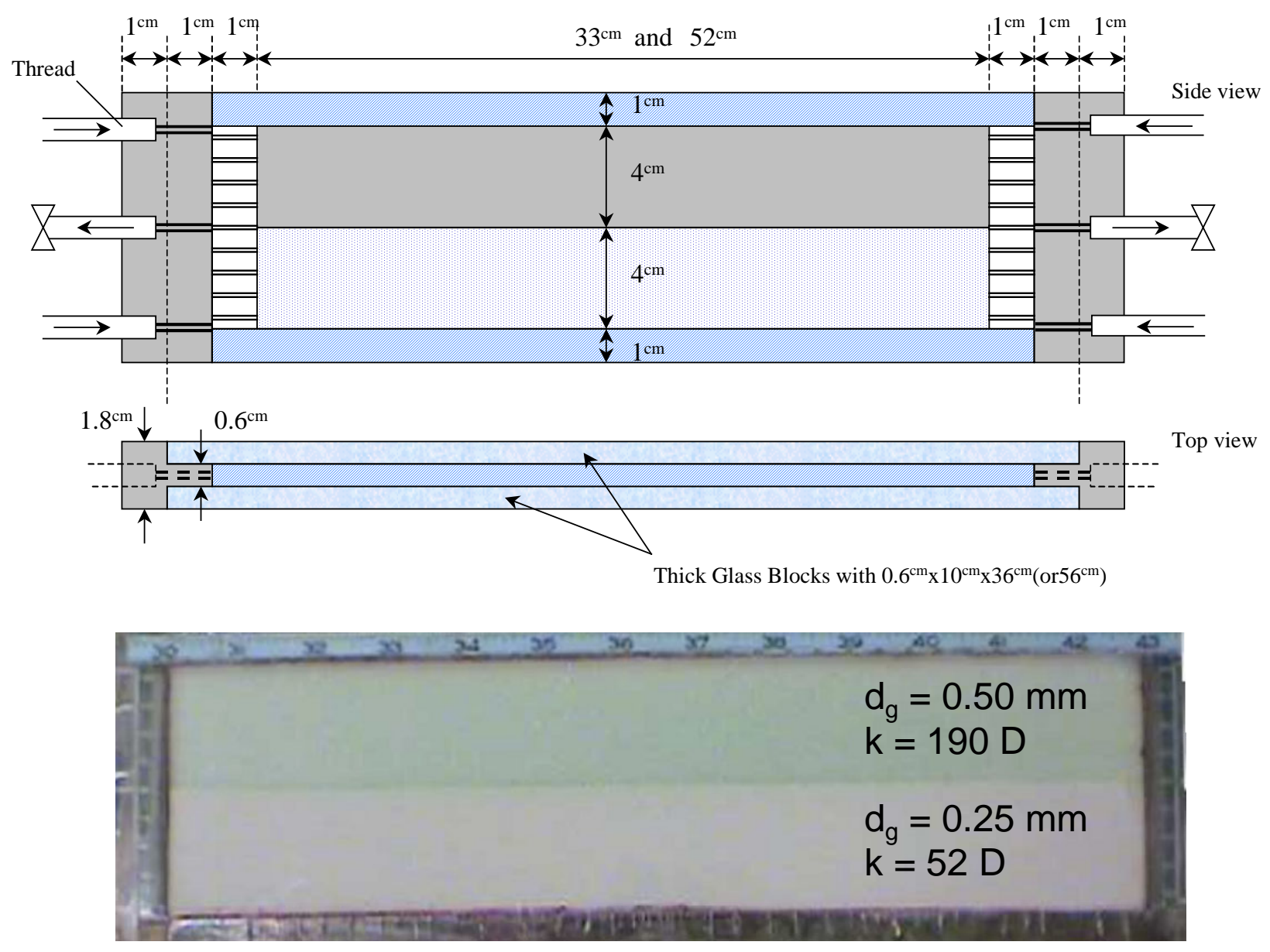

Figure 2 - Glass bead flow model for the experiments. $\phi=39.5 \%$, Permeability ratio $=3.7$. 


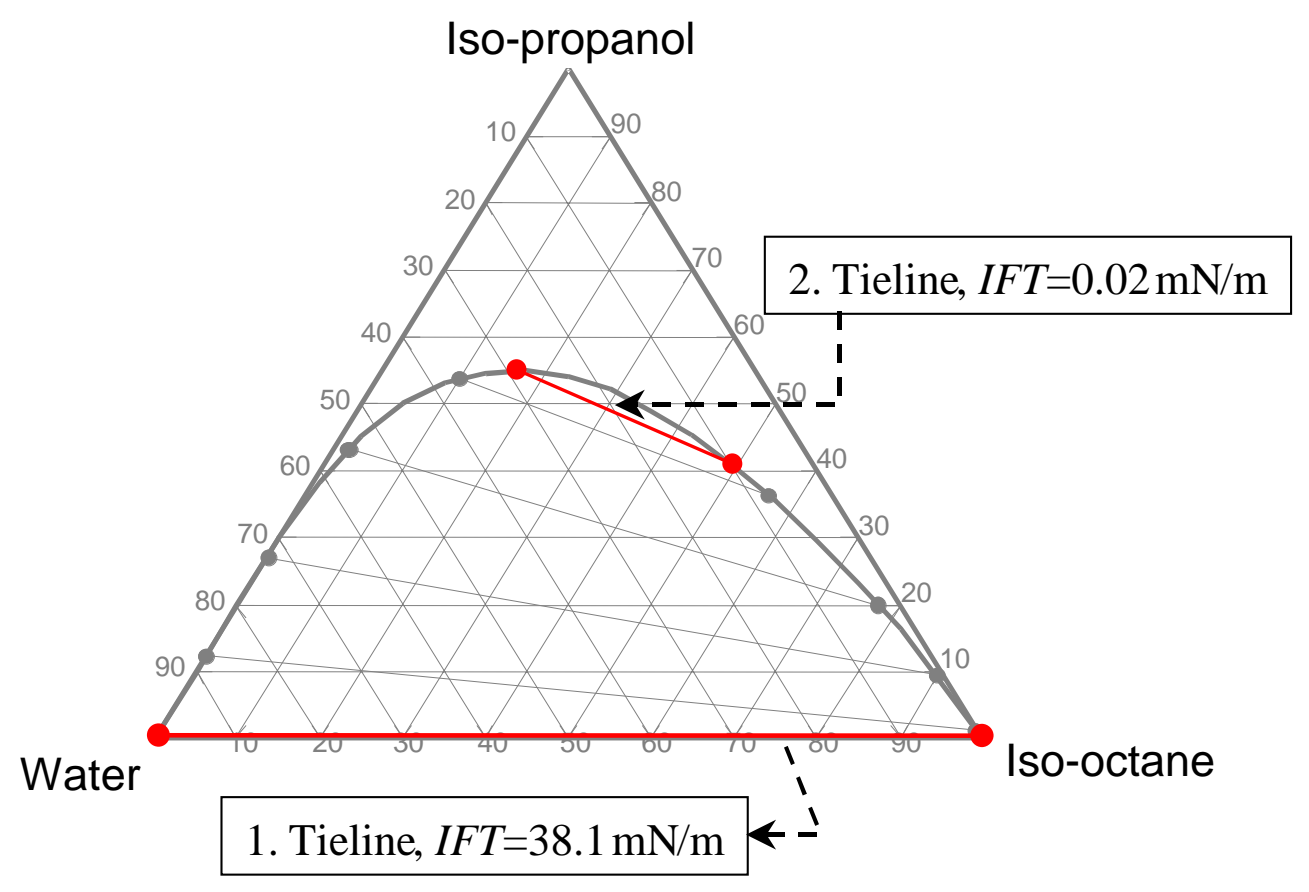

Figure 3 - Fluid system used in the experiments.

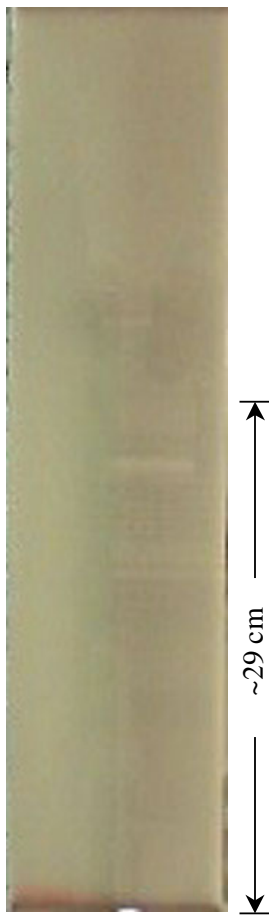

$\mathbf{P V I}=\mathbf{0 . 0}$
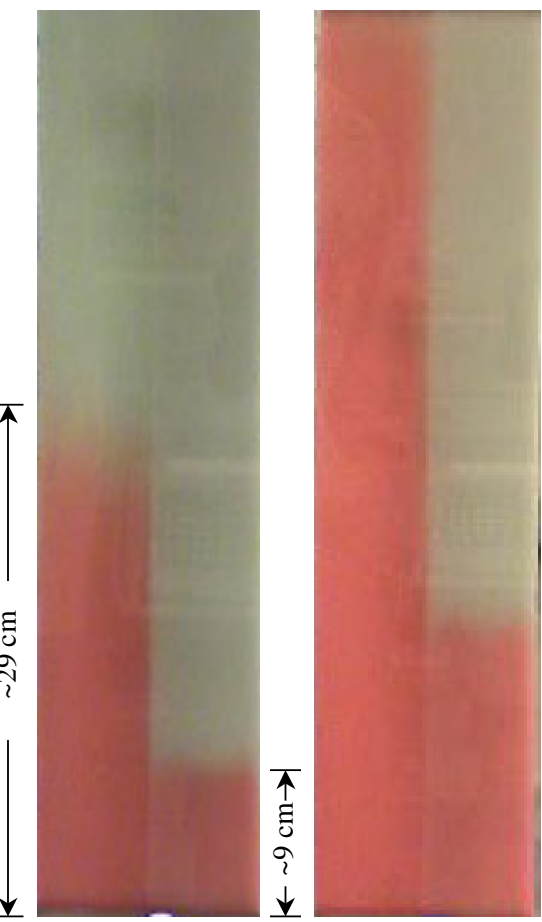

PVI=0.35 At breakthrough

Figure 4 - First contact miscible displacement of water by dyed water $(M=1)$. 


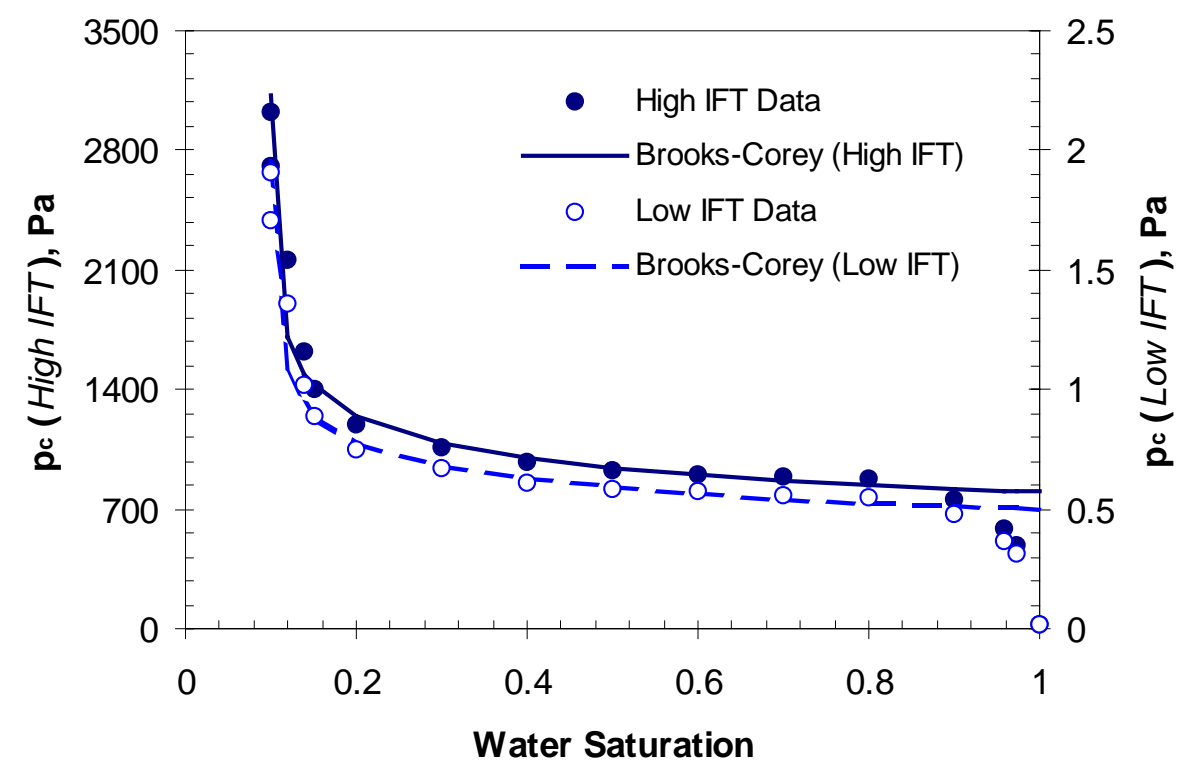

Figure 5 - Brooks-Corey correlation for capillary pressure data for the calculation of the characteristic capillary pressure.

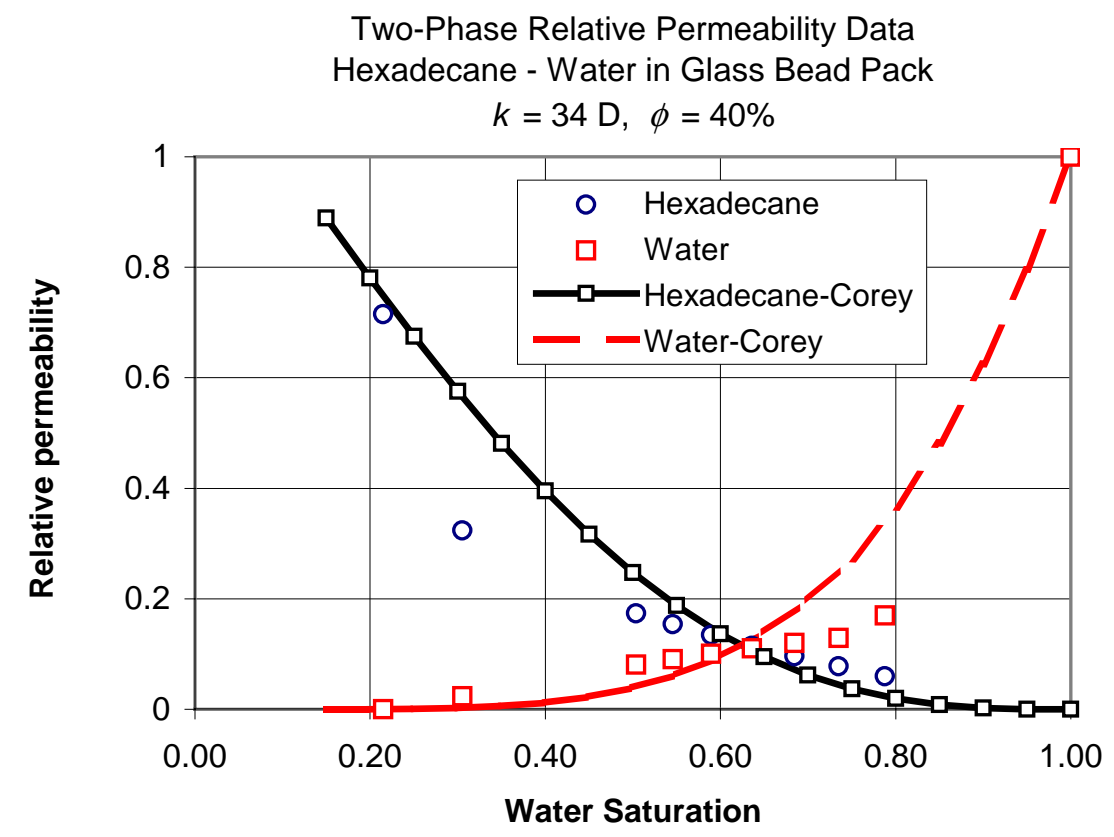

Figure 6 - Relative permeability for the simulation. 


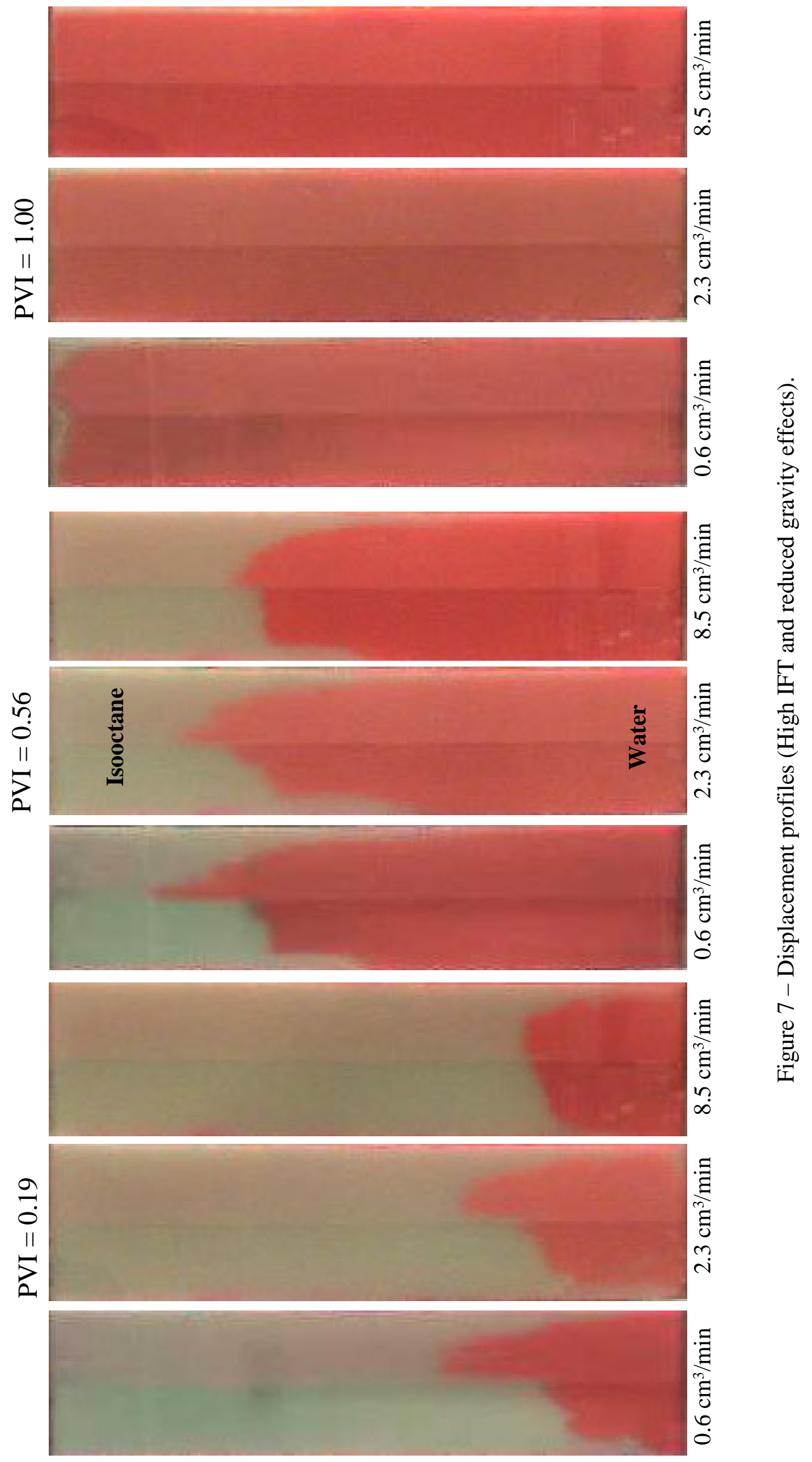



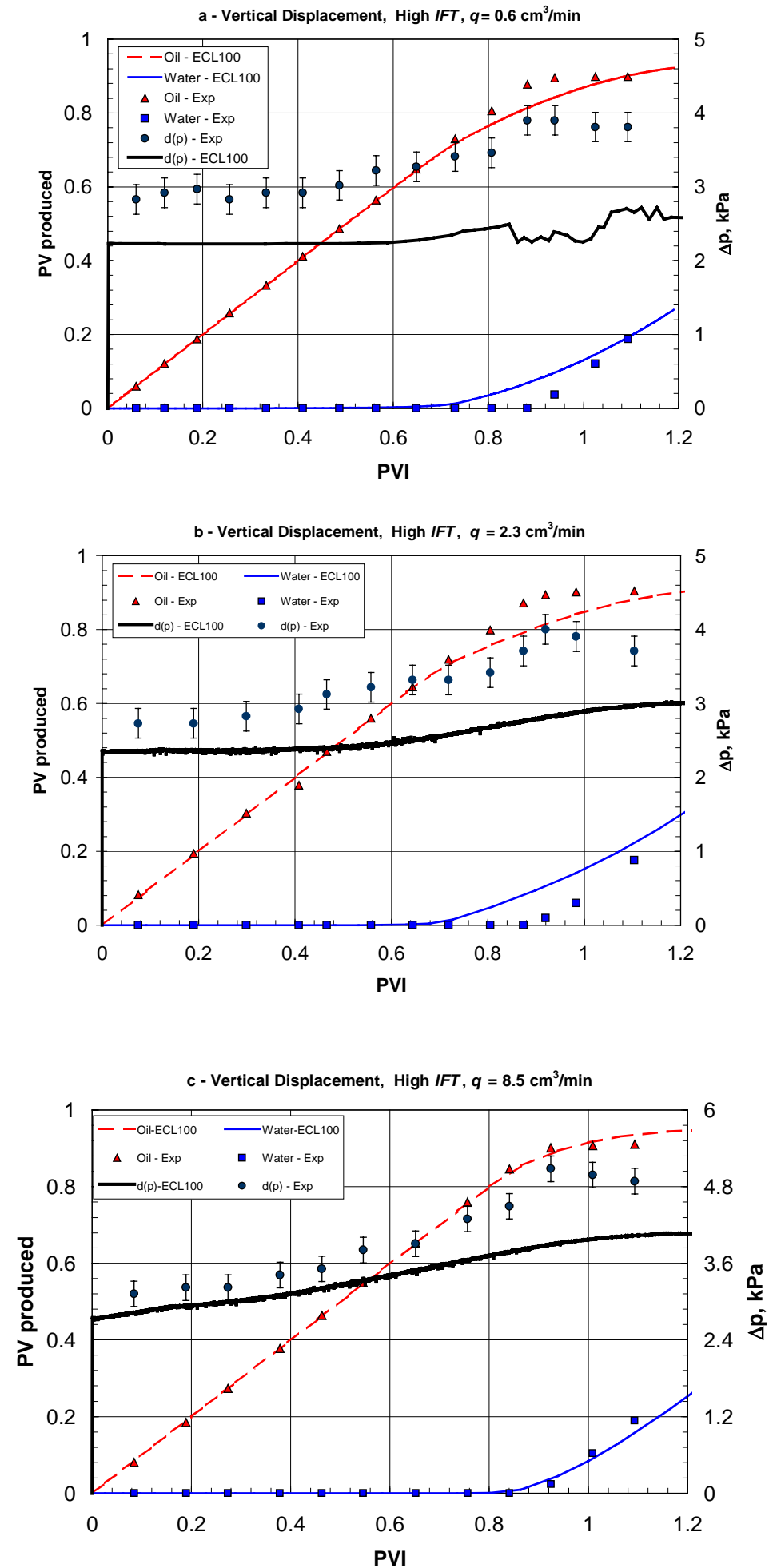

Figure 8 - Production and pressure profiles (High IFT and reduced gravity effects). 


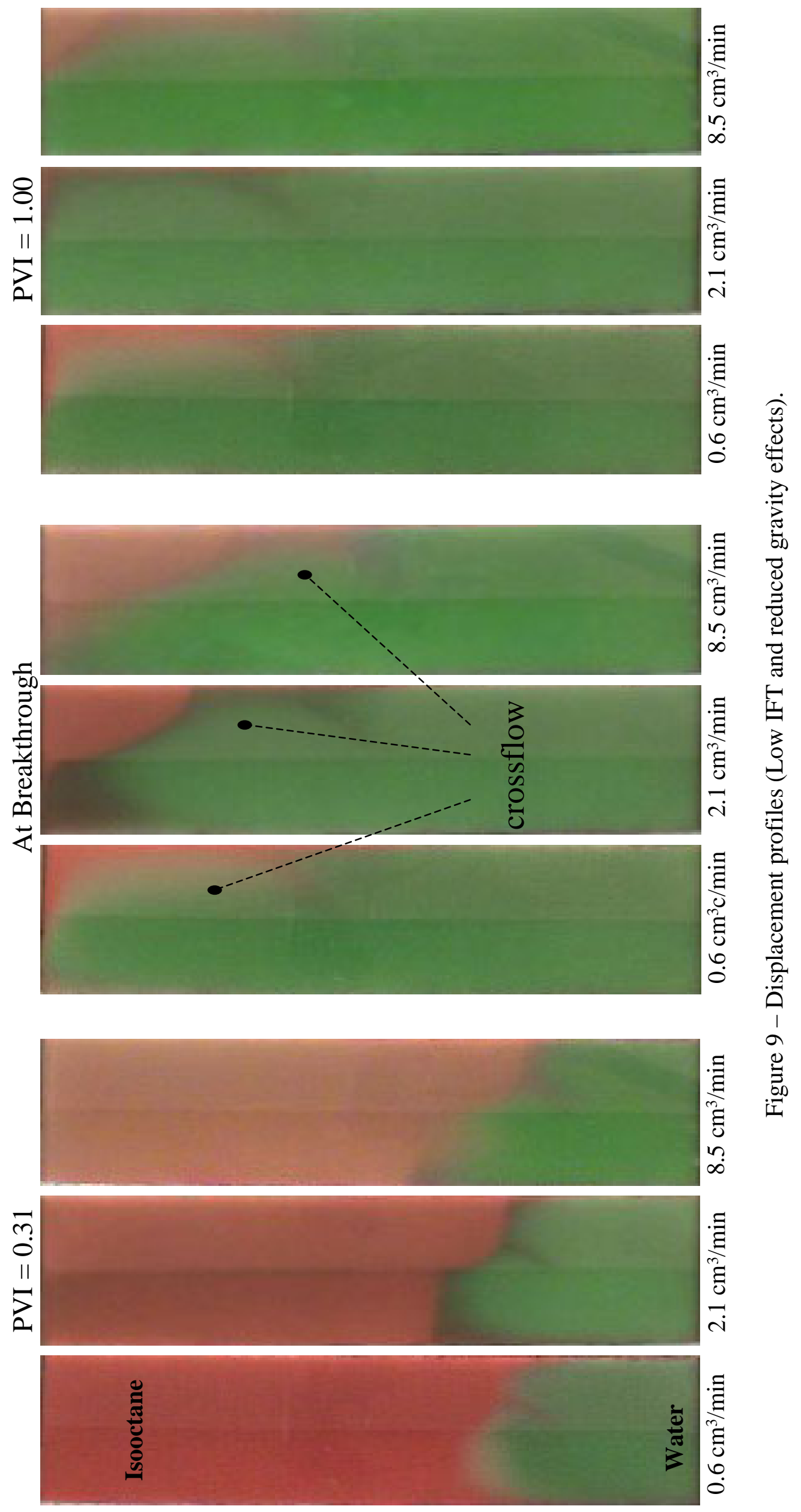




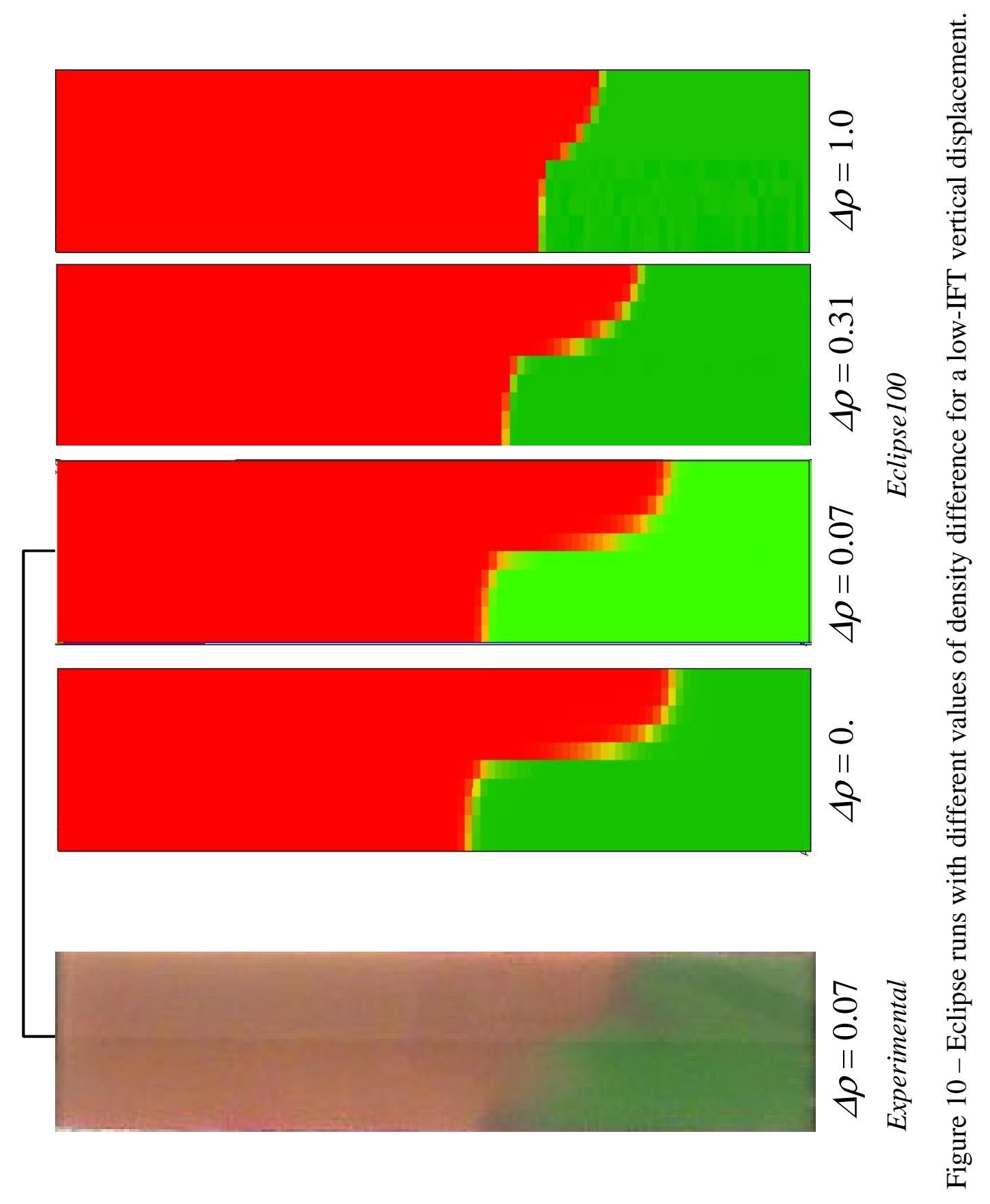


$q=8.5 \mathrm{~cm}^{3} / \mathrm{min}$ at $P V I=0.31$

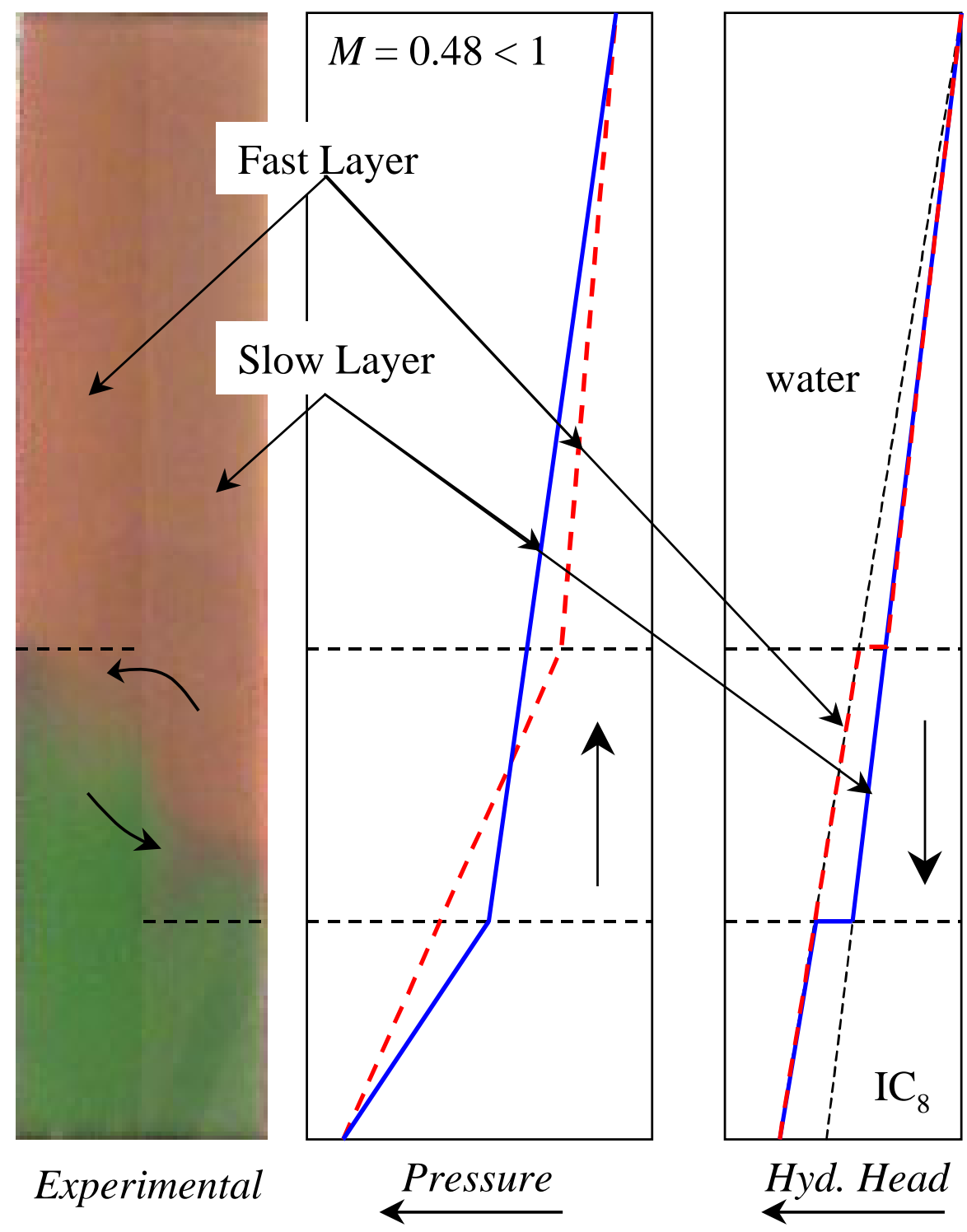

Figure 11 - A schematic of pressure profiles in a low-IFT vertical displacement. 


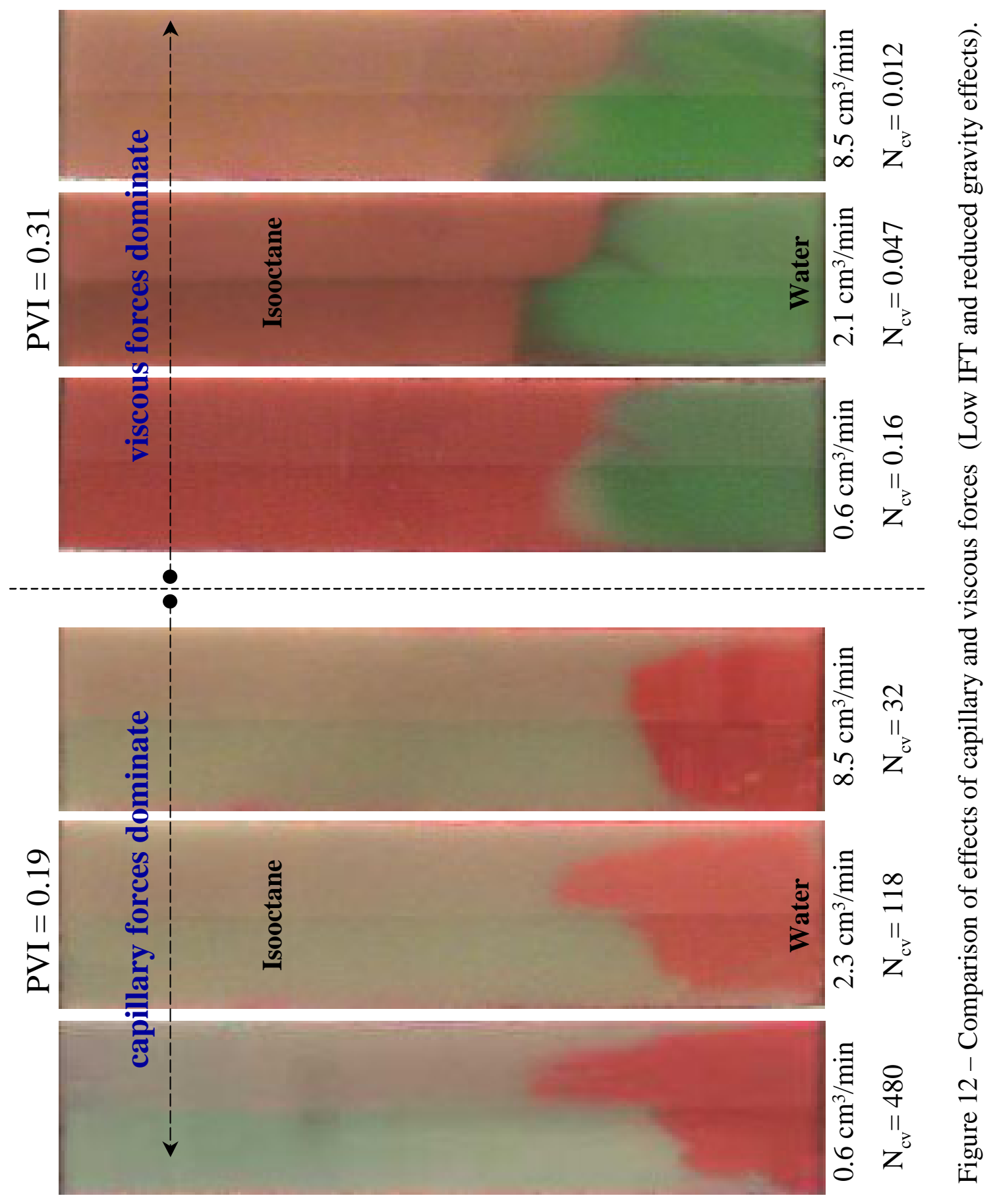



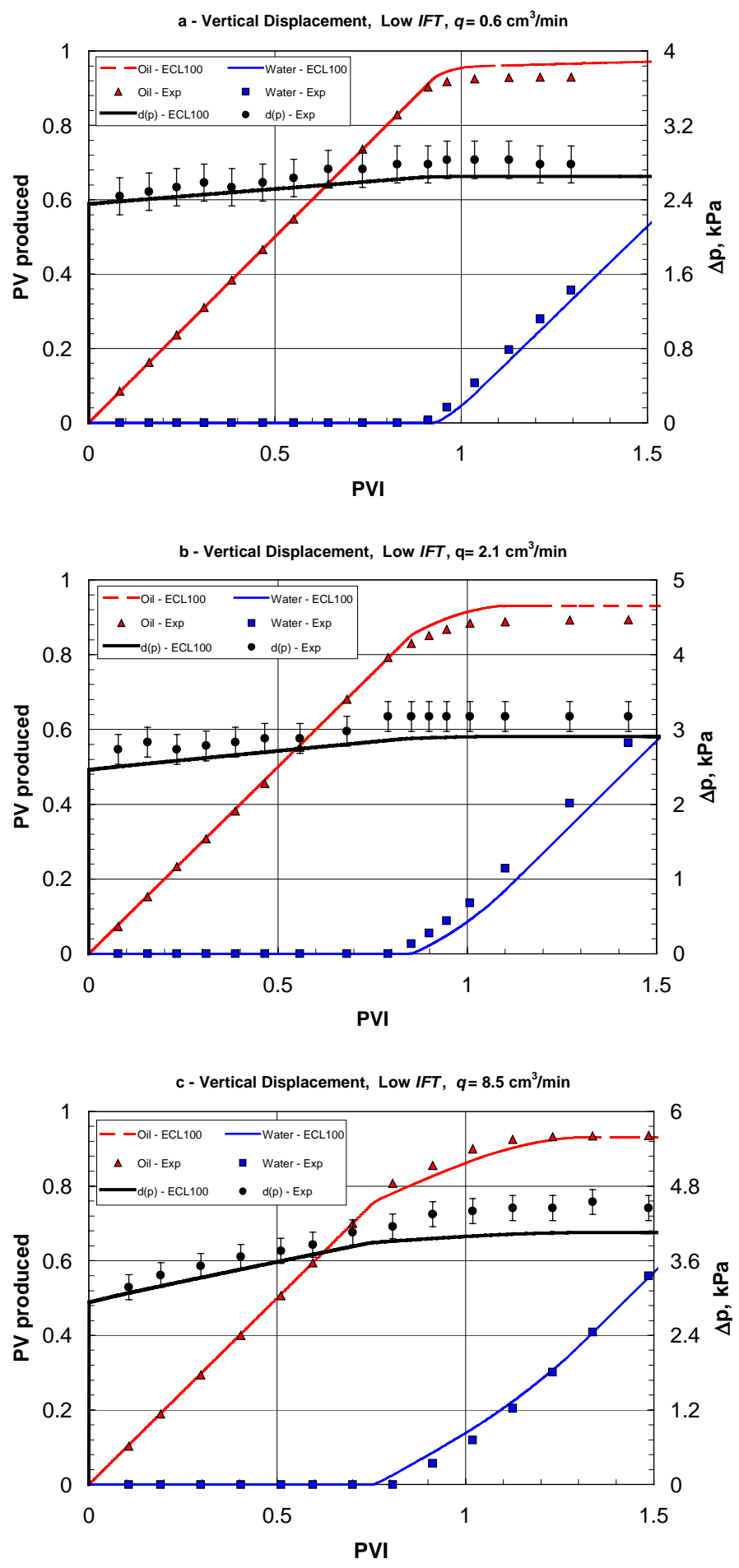

Figure 13 - Production and pressure profiles (Low IFT and reduced gravity effects). 


$$
\text { PVI }=0.20
$$

$0.6 \mathrm{~cm}^{3} / \mathrm{min}$

\section{$2.2 \mathrm{~cm}^{3} / \mathrm{min}$}

$8.9 \mathrm{~cm}^{3} / \mathrm{min}$

$$
\mathrm{PVI}=0.49
$$

$0.6 \mathrm{~cm}^{3} / \mathrm{min}$

$2.2 \mathrm{~cm}^{3} / \mathrm{min}$
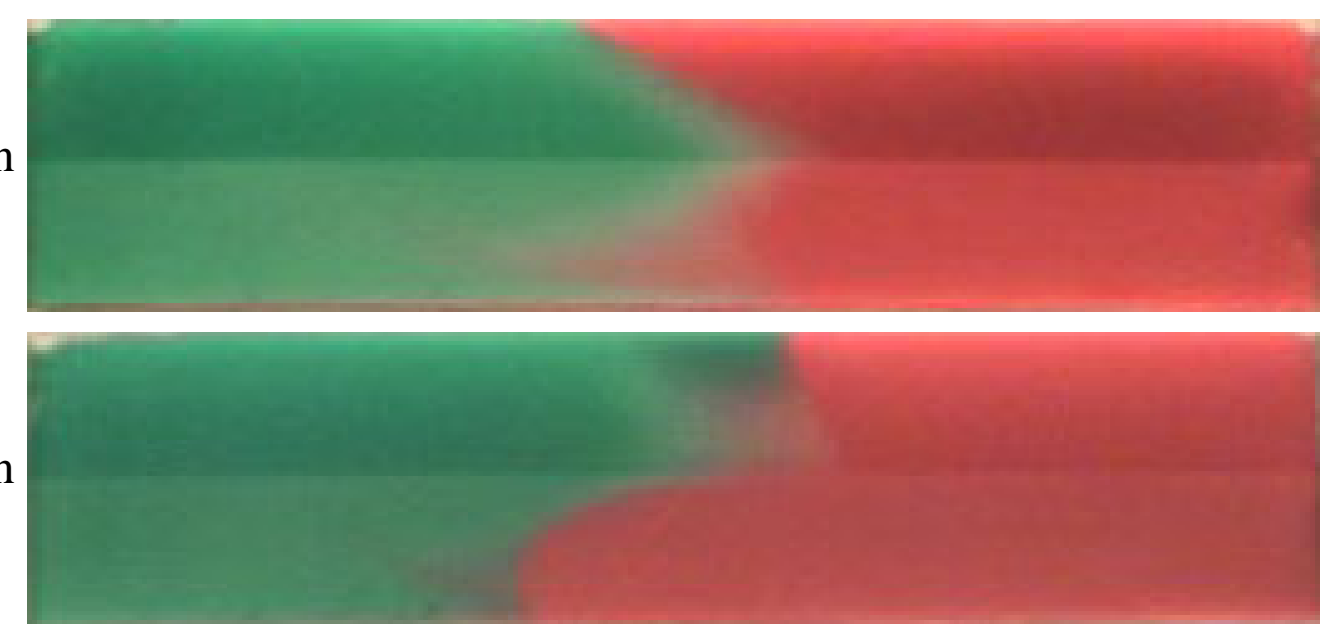

$8.9 \mathrm{~cm}^{3} / \mathrm{min}$

Figure 14 - Saturation profiles (Low IFT and gravity effects). 


\section{$\mathrm{PVI}=0.81$}

$0.6 \mathrm{~cm}^{3} / \mathrm{min}$

$2.2 \mathrm{~cm}^{3} / \mathrm{min}$

$8.9 \mathrm{~cm}^{3} / \mathrm{min}$

$$
\mathrm{PVI}=1.00
$$

$0.6 \mathrm{~cm}^{3} / \mathrm{min}$

$2.2 \mathrm{~cm}^{3} / \mathrm{min}$

$8.9 \mathrm{~cm}^{3} / \mathrm{min}$

Figure 14 (Cntd). 

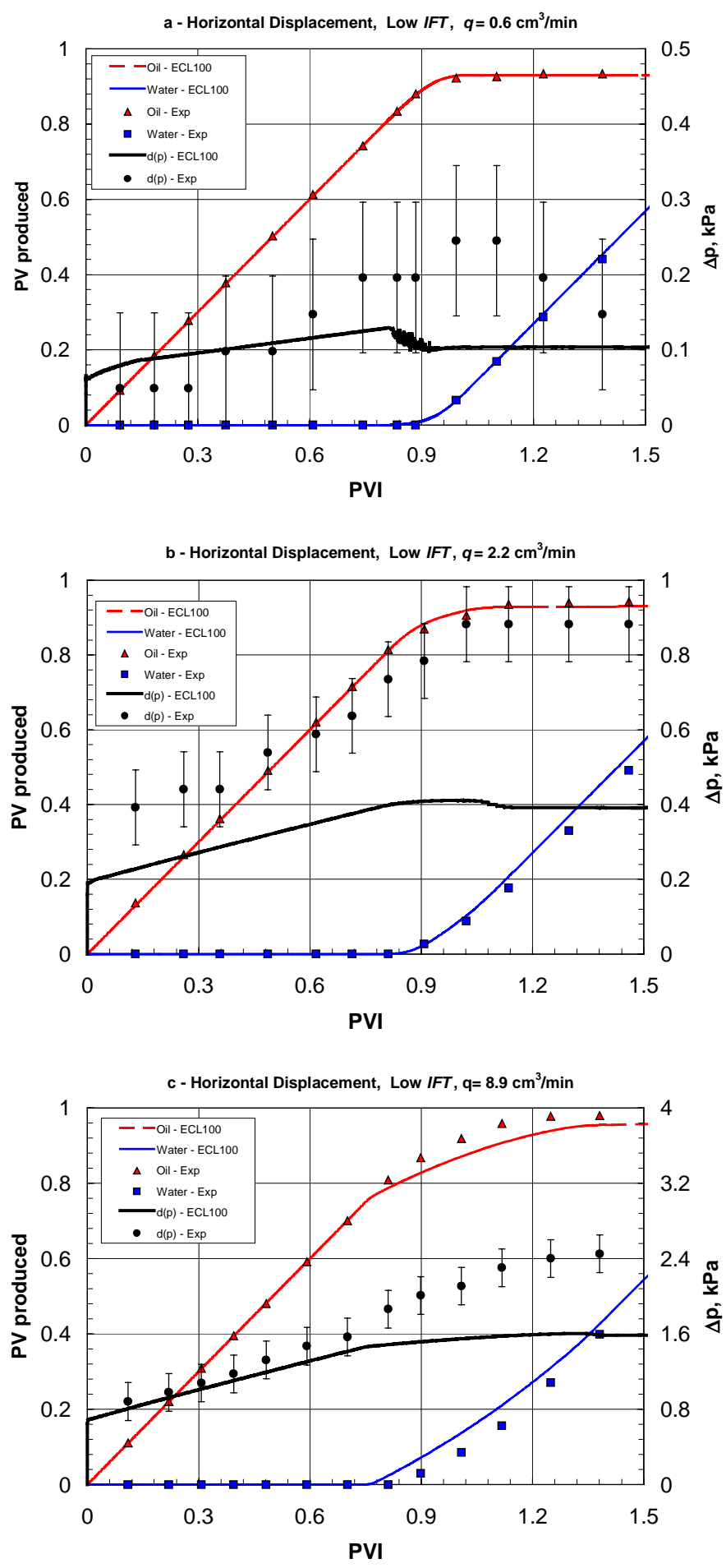

Figure 15 - Production and pressure profiles (Low IFT and gravity effects). 